\title{
5-DESOXIFLAVONÓIDES E LIGNANA ISOLADOS DA MADEIRA DE Schizolobium parahyba (Vell.) S.F. Blake (guapuruvu)
}

\section{Mário Geraldo de Carvalho* e Ildomar Alves do Nascimento}

Departamento de Química, Instituto de Ciências Exatas, Universidade Federal Rural do Rio de Janeiro, BR 465, km 07, 23890-000 Seropédica - RJ, Brasil

\section{Acácio Geraldo de Carvalho}

Departamento de Produtos Florestais, Instituto de Florestas, Universidade Federal Rural do Rio de Janeiro, BR 465, km 07, 23890-000 Seropédica - RJ, Brasil

Recebido em 29/5/07; aceito em 31/1/08; publicado na web em 31/7/08

\begin{abstract}
5-DEOXYFLAVONOIDS AND LIGNAN ISOLATED FROM THE WOOD OF Schizolobium parahyba (Vell.) S.F. Blake (guapuruvu). Four flavonoids, 3,7,4'-trihydroxyflavanone (garbanzol), 7,4'-dihydroxyflavone, 7,4'-dihydroxy-3'-methoxyflavone (geraldone) and 3,4,7,4'-tetrahydroxyflavane (Rel-2R,3S,4S-leucoguibourtinidine, guibourtacacidine) were isolated from the wood of Schizolobium parahyba (Leg., Caesalpinoideae) together with lignan balonofonine, sitosterol, and stigmasterol. The structures were established by IR, NMR and mass spectral data analysis and comparison with literature values.
\end{abstract}

Keywords: Schizolobium parahyba; guapuruvu; 5-deoxyflavonoids.

\section{INTRODUÇÃO}

A espécie vegetal Schizolobium parahyba (Vell.) S.F. Blake, [Leg., Caesalpinoideae], tem sinonímia botânica como Schizolobium excelsum Vog., S. parahybum, S. amazonicum Huber ex Ducke, Caesalpinia parahyba (Vell.) e Cassia parahyba (Vell). ${ }^{1}$ A mesma descrição botânica é usada para a denominação $S$. parahybae (Vell.) S.F. Blake, Schizolobium glutinosum Tul. e Schizolobium kellermanii Pittier. ${ }^{2,3}$ É uma espécie arbórea conhecida popularmente como guapuruvu. Sua distribuição geográfica estende-se do estado da Bahia até Santa Catarina, na floresta pluvial da Mata Atlântica. ${ }^{3}$ Os trabalhos sobre estudo químico de espécies deste gênero relatam apenas a presença de polissacarídeos em exemplares que, de acordo com a região de ocorrência no Brasil, possuem nome popular diferente como guapuruvu (S. parahybae) e pinho cubano (S. amazonicum Ducke). ${ }^{4-6}$ Estes polissacarídeos foram isolados do endosperma das sementes destas plantas e são conhecidos como galactomananas, ${ }^{4}$ xantanas, ${ }^{5}$ arabinanas $^{6}$ e mananas. ${ }^{6}$

Considerando que: não há estudos sobre isolamento e caracterização de metabólitos especiais do guapuruvu; é uma planta que pertence a uma família cujas espécies elaboram substâncias promissoras de atividade biológica; é uma espécie de fácil cultivo e que está sendo feita uma correlação da influência de metabólitos especiais com a resistência de sua madeira ao ataque de Scolytidae, ${ }^{7}$ resolveu-se realizar o estudo fitoquímico desta espécie vegetal. ${ }^{8}$

Este é o primeiro relato de esteróides, lignana e flavonóides neste gênero, assim como o primeiro registro dos dados de RMN da Rel-2$\mathrm{R}, 3 \mathrm{~S}, 4 \mathrm{~S}$-leucoguibourtinidina. A única divulgação de resultados deste estudo foi o completo assinalamento dos dados de $\mathrm{RMN}{ }^{1} \mathrm{H}$ e ${ }^{13} \mathrm{C}$ do garbanzol. ${ }^{9}$ Neste trabalho descrevem-se os primeiros resultados sobre isolamento e identificação de metabólitos especiais desta espécie.

Este estudo permitiu observar que o gênero Schizolobium é bioprodutor de grupo raro de flavonóides, os 5-desoxiflavonóides, que são considerados como marcadores na quimiotaxonomia de leguminosas, ${ }^{10}$ além de perceber a semelhança no metabolismo de

\footnotetext{
*e-mail: mgeraldo@ufrrj.br
}

espécies de Guibourtia e Schizolobium da subfamíla Caesalpinoideae. Outra consideração relevante em relação a este grupo de flavonóides é a propriedade de auxiliarem no processo de fixação de nitrogênio, justificando, inclusive, a utilização de leguminosas como adubo verde na agricultura. ${ }^{11}$

\section{RESULTADOS E DISCUSSÃO}

O fracionamento cromatográfico e análise das frações dos extratos da madeira de $S$. parahyba (ou $S$. parahybae) conduziram ao isolamento e identificação de uma mistura constituída de sitosterol (1) e estigmasterol (1a), da 3,3'-dimetoxi-4', 9' -diidroxi-(4-O7',5-8')-lignana-7,8-deidro-9-al (balonofonina, 2) e dos flavonóides, 3,7,4'-triidroxiflavanona (garbanzol, 3), 7,4'-diidroxiflavona (4), 7,4'-diidroxi-3' '-metoxiflavona (geraldona, 5) e a 3,4,7,4'-tetraidroxiflavana (guibourtacacidina, 6).

A mistura das substâncias $\mathbf{1}$ e $\mathbf{1 a}$ foi isolada como um sólido cristalino e identificada através da análise dos espectros de IV e RMN ${ }^{1} \mathrm{H}$ e comparação com dados da literatura. ${ }^{12}$

O espectro de IV da substância 2 apresentou bandas em $3435 \mathrm{~cm}^{-1}$ $\left(v_{\mathrm{O}-\mathrm{H}}\right), 1711\left(\mathrm{v}_{\mathrm{C}=\mathrm{O}}\right)$ e em 1662, 1592, 1515, $1492 \mathrm{~cm}^{-1}\left(\mathrm{v}_{\mathrm{C}=\mathrm{C}}\right), 965$ e 828 $\mathrm{cm}^{-1}\left(\delta_{\mathrm{C}-\mathrm{H}}\right)$ de dupla e de anel aromático. A análise do espectro de RMN ${ }^{1} \mathrm{H}$ desta substância permitiu identificar cinco hidrogênios em anel aromático representados pelos sinais em $\delta_{\mathrm{H}} 6,88(\mathrm{~s}, 3 \mathrm{H}), 7,03(\mathrm{~s}, 1 \mathrm{H})$ e 7,12 (s, 1H), além dos sinais em $\delta_{\mathrm{H}}: 7,40(\mathrm{~d}, J=16,0 \mathrm{~Hz} ; 1 \mathrm{H}), 6,61$ $\left(\mathrm{dd}, J_{1}=16,0 \mathrm{~Hz}\right.$ e $\left.J_{2}=7,7 \mathrm{~Hz} ; 1 \mathrm{H}\right)$ e $9,63(\mathrm{~d}, J=7,7 \mathrm{~Hz} ; 1 \mathrm{H})$ que foram atribuídos a uma enona contendo um aldeído $(-\mathrm{CH}=\mathrm{CH}-\mathrm{CH}=\mathrm{O})$. Os dados obtidos dos espectros de RMN ${ }^{13} \mathrm{C}$ (BBD e DEPT), $\delta_{\mathrm{CH}}: 193,6$, 153,1 e 118,1, confirmaram a presença desta unidade. Além dos sinais compatíveis com a enona, estes espectros apresentaram sinais para cinco carbonos metínicos e sete carbonos quaternários de anel aromático que, de acordo com os valores de deslocamento químico, quatro são oxigenados e três são não oxigenados. Os valores de freqüência dos demais sinais estão compatíveis com carbonos $\mathrm{sp}^{3}$, sendo dois carbonos metínicos $\left(\delta_{\mathrm{CH}}: 88,9\right.$ e 52,9), um metileno ligado a oxigênio $\left(\delta_{\mathrm{CH} 2} 63,9\right)$ e duas metoxilas $\left(\delta_{\mathrm{CH} 3} 56,0\right.$ e 55,9$)$. Os sinais adicionais observados no espectro de $\mathrm{RMN}^{1} \mathrm{H}$ em $\delta_{\mathrm{H}} 5,63(\mathrm{~d}, J=7,2 \mathrm{~Hz}, 1 \mathrm{H}), 3,95(\mathrm{~m}, 2 \mathrm{H})$, 
3,66 (m, 1H), 3,88 (s, $\left.\mathrm{OCH}_{3}\right)$ e 3,92 (s, $\left.\mathrm{OCH}_{3}\right)$ e a análise do espectro ${ }^{1} \mathrm{Hx}{ }^{1} \mathrm{H}$-COSY permitiram propor duas unidades $\mathrm{C}_{6}-\mathrm{C}_{3}$ de uma lignana contendo um anel furânico com um grupo hidroximetileno. $\mathrm{O}$ valor da constante de acoplamento do sinal em $\delta_{\mathrm{H}} 5,63(J=7,2 \mathrm{~Hz})$ permitiu deduzir a relação trans entre os hidrogênios do anel furânico. Os dados de $\mathrm{RMN}$ de ${ }^{13} \mathrm{C}$ e ${ }^{1} \mathrm{H}$ foram comparados com os divulgados para lignanas dibenzofurânicas e observou-se que 2 corresponde à lignana balonofonina [3,3'-dimetoxi-4', 9'-diidroxi-(4-O-7',5-8')-lignana-7,8deidro-9-al]. A possibilidade de localização dos dois grupos metoxilas em um anel foi descartada com base nas seguintes observações: consideração biossintética; a diferença dos deslocamentos químicos dos carbonos divulgados para as lignanas hierochina $\mathrm{A}$, hierochina $\mathrm{B}^{13} \mathrm{e}$ para a americanina $\mathrm{D} ;{ }^{14}$ como exemplo, a localização da metoxila em 4 ' resultaria em um deslocamento químico diferente para CH-2' $\left[\delta_{\mathrm{CH}} 110,5\right.$ (2) e 113,1 (americanina D)]. ${ }^{14}$ Essas considerações permitiram propor para 2 a estrutura da Rel-7'R,8'R-3,3'-dimetoxi-4',9'-diidroxi-(4-O7',5-8')-lignana-7,8-deidro-9-al, isolada de Balonophora japonica ${ }^{15} \mathrm{e}$ de Chosenia arbutifolia. ${ }^{16}$

A substância 3 foi identificada como o flavanonol, 3,7,4'-triidroxiflavanona, através da análise dos espectros de RMN 1D e 2D conforme descrito por Nascimento e colaboradores ${ }^{9}$ e comparação dos valores divulgados para a aglicona do 3-O-glucopiranosil-garbanzol (lecontina), isolada de Baptisia lecontei (Leguminosae). ${ }^{17}$ Desta forma, 3 foi identificada como o garbanzol, isolado anteriormente de várias espécies de Leguminosae como Cicer arietinum, ${ }^{18}$ Onobrychis viciifolia,${ }^{19}$ Trifolium subterraneum,,${ }^{20}$ Pterocarpus marsupium ${ }^{21}$ e de Rhus succedanea L. (Anacardiaceae). ${ }^{22}$ Esse é o primeiro registro do isolamento e identificação do garbanzol em Caesalpinoideae.

Conforme descrito na parte experimental, as substâncias $\mathbf{4}$ e $\mathbf{5}$ foram obtidas puras e tiveram seus respectivos espectros de RMN ${ }^{1} \mathrm{H}$ e ${ }^{13} \mathrm{C}$ analisados separadamente. Verificou-se que ambas seriam 5-deoxiflavonas com diferença apenas no padrão de substituição do anel B. Os espectros de IV das duas substâncias apresentaram sinais compatíveis com fenóis e carbonila conjugada. Os espectros de RMN ${ }^{1} \mathrm{H}$ apresentaram um dupleto $(J=8,8 \mathrm{~Hz}, \mathrm{H}-5)$ em campo baixo $\left[\delta_{\mathrm{H}}\right.$ $7,86(4)$ e $7,78(5)]$ que, de acordo com os sinais do espectro ${ }^{1} \mathrm{Hx}{ }^{1} \mathrm{H}-$ COSY, acoplam com o duplo dupleto $\left(J_{1}=8,8\right.$ e $\left.J_{2}=2,2 \mathrm{~Hz}, \mathrm{H}-6\right)$ em $\delta_{H} 6,91(4)$ e $6,69(5)$ e estes com o dupletos $(J=2,2 \mathrm{~Hz}, \mathrm{H}-8)$ em $\delta_{\mathrm{H}} 6,67(\mathbf{4}), 6,62(5)$, respectivamente. Estes dados e a ausência de sinal acima de 12,5 ppm (hidroxila quelada) permitiram propor para ambas um anel A de uma flavona com oxigenação em 7. A presença de um simpleto adicional em $\delta_{\mathrm{H}} 6,72$ (4) e 6,53 (5), atribuídos ao $\mathrm{H}-3$, confirmaram as propostas de flavonas. Os espectros de RMN ${ }^{1} \mathrm{H}$ (1D e 2D) da substância 4 apresentaram sinais compatíveis com um sistema AA'BB' $\left[\delta_{\mathrm{H}} 7,91(\mathrm{~J}=8,8 \mathrm{~Hz}, 2 \mathrm{H}, \mathrm{H}-2\right.$ ', 6 ' $)$ e $6,92(J=8,8$ $\left.\mathrm{Hz}, 2 \mathrm{H}, 3^{\prime}, 5^{\prime}\right]$. Isso permitiu propor para 4 a estrutura da $7,4^{\prime}$-diidroxiflavona que, inclusive, está de acordo com os dados observados nos espectros de RMN ${ }^{13} \mathrm{C}$ (BBD e DEPT). Esses dados foram idênticos aos registrados na literatura para esta flavona isolada de Piptadenia rigida ${ }^{23}$ e Mendicago sativa. ${ }^{24}$ Esta substância foi isolada, também, de cultura de células de Glycyrrhiza echinata ${ }^{25}$ e de espécies de Baptisia (Leguminosae) ${ }^{26}$. A análise dos demais sinais dos espectros de RMN ${ }^{1} \mathrm{H}$ e ${ }^{1} \mathrm{Hx}{ }^{1} \mathrm{H}-\mathrm{COSY}$ de 5 permitiu perceber que a diferença entre $\mathbf{4} \mathrm{e}$ 5 estava no padrão de substituição do anel B. Além dos três sinais adicionais de hidrogênios de sistema aromáticos $\left[\delta_{\mathrm{H}} 7,56\left(\mathrm{dd}, J_{1}=\right.\right.$ 8,4 e $J_{2}=2,2 \mathrm{~Hz}, 1 \mathrm{H}, \mathrm{H}-6$ ') $, 7,43$ (d, $J=2,2,1 \mathrm{H}, \mathrm{H}-2^{\prime}$ ), 6,85 (d, $J=$ $\left.8,4 \mathrm{~Hz}, 1 \mathrm{H}, \mathrm{H}-5^{\prime}\right)$ ] observou-se um sinal de um grupo metoxila em $\delta_{\mathrm{H}}$ 3,93 (s, 3H). O espectro 2D-NOESY apresentou sinais de interação espacial dos hidrogênios H-3 $\left(6,53\right.$, s) e $\mathrm{OCH}_{3}(3,93$, s) com o H-2' $(7,43, d)$. Com essas informações e os valores dos picos detectados no EM em $m / z 284\left(\mathrm{M}^{+}, \mathrm{C}_{16} \mathrm{H}_{12} \mathrm{O}_{5}\right), 254$ (95), 226 (48), 137 (100\%), definiu-se a estrutura de 5 como 7,4'-diidroxi-3'-metoxiflavona cujos dados de RMN foram idênticos aos divulgados por Jung e co-

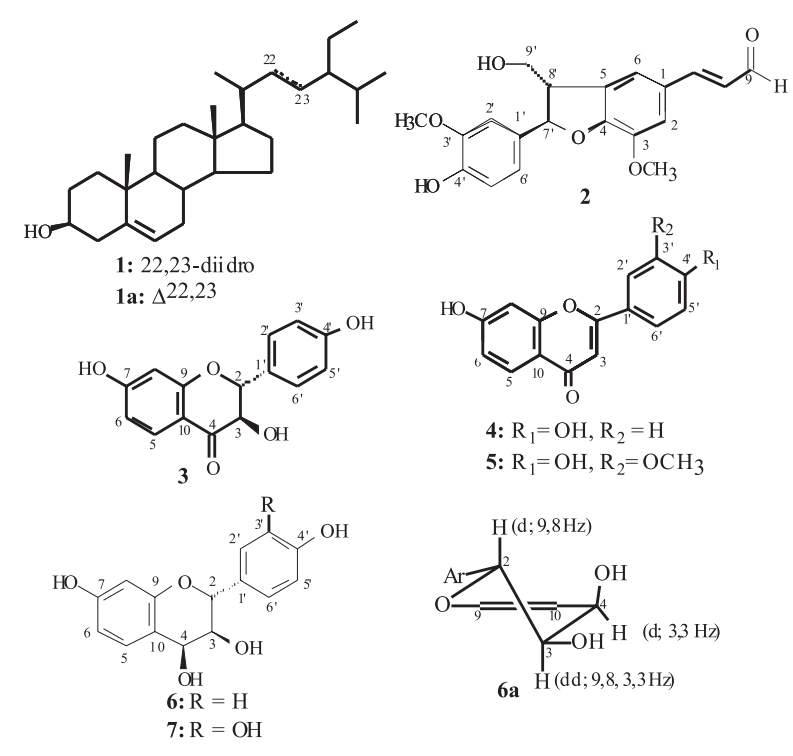

laboradores. ${ }^{27}$ Esta substância é conhecida como geraldona, isolada também de Trifolium subterraneum,${ }^{20}$ T. repens,${ }^{13}$ Vicia $f a b a^{28} \mathrm{e}$ de Albizzia julibrissin. ${ }^{27}$

$\mathrm{O}$ espectro de $\mathrm{RMN}{ }^{1} \mathrm{H}$ de $\mathbf{6}$ apresentou cinco sinais na região de deslocamento químico de hidrogênios de anel aromático. Os dupletos em $\delta_{\mathrm{H}} 7,25(J=8,5 \mathrm{~Hz}, 2 \mathrm{H})$ e $6,77(\mathrm{~J}=8,5 \mathrm{~Hz}, 2 \mathrm{H})$ foram atribuídos aos hidrogênios de um sistema AA'BB'. O simpleto largo em $\delta_{\mathrm{H}} 6,23(\mathrm{sl}, 1 \mathrm{H})$ e os sinais em $6,37(\mathrm{dl}, J=8,0 \mathrm{~Hz} ; 1 \mathrm{H})$ e $7,10(\mathrm{~d}$, $J=8,0 \mathrm{~Hz}, 1 \mathrm{H}$ ) são compatíveis com um sistema ABC de outro anel aromático. Os demais sinais em $\delta_{\mathrm{H}} 4,98(\mathrm{~d}, J=9,8 \mathrm{~Hz}, 1 \mathrm{H}), 4,56$ (d, $J=3,3 \mathrm{~Hz} ; 1 \mathrm{H})$ e $3,87\left(\mathrm{dd}, J_{1}=9,8 \mathrm{~Hz}\right.$ e $\left.J_{2}=3,3 \mathrm{~Hz}, 1 \mathrm{H}\right)$ representam três hidrogênios vizinhos ligados a carbonos oxigenados. A análise dos sinais observados no espectro de $\mathrm{RMN}{ }^{1} \mathrm{Hx}{ }^{1} \mathrm{H}-\mathrm{COSY}$ permitiu confirmar os acoplamentos entre os hidrogênios dos respectivos sistemas propostos. Os espectros de RMN ${ }^{13} \mathrm{C}$ (BBD e DEPT) desta substância apresentaram, além dos sinais relativos aos carbonos do anel aromático, três sinais na região de carbonos metínicos $\mathrm{sp}^{3}$ ligados a oxigênio [ $\delta_{\mathrm{CH}} 79,1,73,5$ e 69,1$]$. Esses dados estão de acordo com uma 3,4-dioxi-flavana e a comparação com dados da literatura para as leucoguibourtinidinas, ${ }^{29}$ permitiu propor para 6 a estrutura $3,4,7,4^{\prime}$ tetraidroxiflavana. A configuração relativa (2,3-trans-3,4-cis) como representada em 6a é compatível com os valores das constantes de acoplamento calculadas a partir dos dados do espectro de RMN ${ }^{1} \mathrm{H}$ (400 MHz, $\left.\mathrm{D}_{3} \mathrm{COD}\right)$. Não foram encontrados dados de RMN desta estrutura e, por isso, compararam-se os valores de 6 com os divulgados para o $4 \beta$-fisetinidol [7, em $\left.\left(\mathrm{D}_{3} \mathrm{C}\right)_{2} \mathrm{CO}+5 \% \mathrm{D}_{2} \mathrm{O}\right] \cdot{ }^{30}$ Esta substância corresponde à Rel-2R,3S,4S-3,4,4',7-tetraidroxiflavana (guibourtacacidina), isolada de Guibourtia coleosperma ${ }^{29}$ e de Musa sapientium L. (Musaceae). ${ }^{31} \mathrm{~A}$ completa atribuição dos deslocamentos químicos de hidrogênio e carbono-13 de $\mathbf{6}$ é descrita neste trabalho.

\section{PARTE EXPERIMENTAL}

\section{Procedimentos experimentais gerais}

Os espectros na região do infravermelho foram obtidos em espectrômetro Perkin-Elmer 1600/1605 FT-IR usando pastilha com KBr ou filme em placas de $\mathrm{NaCl}$. Os espectros de ressonância magnética nuclear ${ }^{1} \mathrm{H} \mathrm{e}{ }^{13} \mathrm{C}$ (incluindo experimentos 2D) foram obtidos em espectrômetros Bruker ACE-200 [200 MHz $\left({ }^{1} \mathrm{H}\right)$ e $\left.50 \mathrm{MHz}\left({ }^{13} \mathrm{C}\right)\right]$ e Jeol Elipse JNM-GX-400 [400 MHz $\left({ }^{1} \mathrm{H}\right)$ e $\left.100 \mathrm{MHz}\left({ }^{13} \mathrm{C}\right)\right]$. Como padrão interno para referência de deslocamento químico foi usado 
tetrametilsilano. Foi usada cromatografia em coluna de sílica gel (230-400 e 70-230 mesh) da Vetec ou Aldrich como fase estacionária. A cromatografia em camada delgada preparativa (CCP) foi feita em placas de sílica gel $60 \mathrm{PF}_{254}$, Vetec, sobre suporte de vidro e espessura de $1 \mathrm{~mm}$ e reveladas com irradiação ultravioleta de $\lambda=254$ ou 365 $\mathrm{nm}$. Foram usadas placas de sílica gel $60 \mathrm{PF}_{254}$ em alumínio (Sorbent) para análise cromatográfica em camada fina $(\mathrm{CCF})$ e como reveladores foram usados, além da detecção por radiação ultravioleta ( $\lambda 254$ e 366 $\mathrm{nm}$ ), vapores de iodo, reagentes: Liebermann-Burchard; sulfato cérico $(1 \%)+\mathrm{H}_{2} \mathrm{SO}_{4}(10 \%)$ e soluções $1 \%$ de $\mathrm{AlCl}_{3}$ em etanol.

\section{Material vegetal}

A madeira de Schizolobium parahyba (ou S. parahybae) foi coletada no campus da Universidade Federal Rural do Rio de Janeiro, no Instituto de Florestas, em abril de 2000, cuja exsicata está depositada no herbário RBR (№ 1171), Dpto. de Botânica-IB-UFRRJ, identificada pela $D^{\text {ra }}$ M. de M. S. Conde (IB-UFRRJ). Este material tem sido usado pelo Dr. A. G. de Carvalho (IF-UFRRJ) como parte do projeto "Suscetibilidade da madeira de essências arbóreas a Scolytidae e Platypodidae (Coleoptera)". ${ }^{7}$

\section{Extração e isolamento dos constituintes químicos}

A madeira, após seca e moída (1650,0 g), foi submetida à maceração exaustiva com diclorometano e metanol, consecutivamente, a temperatura ambiente. Os extratos foram concentrados sob pressão reduzida em evaporador rotativo, obtendo-se os extratos EGD (14,0 g) e (EGM, 50,0 g), respectivamente. O extrato obtido com diclorometano (EGD, 10,0 g) foi fracionado em coluna com gel de sílica usando $\mathrm{CH}_{2} \mathrm{Cl}_{2}$ como solvente inicial e mistura com acetato de etila em ordem crescente de polaridade e recolhidas 100 frações de $50 \mathrm{~mL}$. Um material sólido obtido das frações 30-34 foi recristalizado em metanol e forneceu 1+1a (170,0 mg). As frações 36-44 foram reunidas e submetidas à cromatografia em coluna flash de gel de sílica usando mistura de diclorometano com acetato de etila e as subfrações 35-43 obtidas com a relação 7:3 dessa mistura forneceram 2 (óleo, 28,0 mg).

Seguindo os procedimentos semelhantes aos de Pauletti e colaboradores, ${ }^{32}$ o extrato metanólico (EGM, 45,0 g) foi dissolvido em metanol:água (7:3) e adicionado acetato de etila (3x $200 \mathrm{~mL})$. A parte solúvel em acetato de etila foi concentrada à pressão reduzida e após completa retirada do solvente forneceu 25,0 g de resíduo codificado como EGMAC. Este material foi pulverizado com sílica de granulação adequada para cromatografia em coluna e aplicada em uma coluna de gel de sílica e como fase móvel foram utilizados: hexano e acetato de etila com 30, 50, 70 até 100\% de acetato de etila. Recolheram-se 112 frações $(250 \mathrm{~mL})$ com estas misturas e, então, mais 4 frações $(500 \mathrm{~mL}$ ) usando metanol 100\%. Fez-se triagem para identificar as frações mais fáceis de purificar levando-se em consideração a melhor separação com diferentes sistemas de eluentes em cromatografia em camada fina. Obtiveram-se os grupos de frações: EGMAC-18-21 (65,0 mg), EGMAC-30-41 (1,12 mg) e EGMAC-72-85 (1,2 g). O grupo EGMAC-18-21 foi submetido à cromatografia de camada delgada preparativa e foi eluída três vezes na mistura $\mathrm{CHCl}_{3}: \mathrm{CH}_{3} \mathrm{OH}(7: 3)$. A placa foi observada na luz UV $\left(v_{\text {máx }} 254 \mathrm{~nm}\right)$ e separou-se a faixa que apresentou coloração amarela. A substância foi extraída da sílica mediante filtração em funil de Hirsch, utilizando-se acetato de etila e metanol, e obteve-se um material pastoso que foi identificado como 3 (20,0 mg). Após a evaporação parcial do solvente do grupo EGMAC-30-41 houve a formação de um precipitado amarelo, insolúvel em acetato de etila e acetona e pouco solúvel em metanol. Filtrou-se o precipitado em funil de Hirsch lavando-o com acetato de etila. O sólido foi identificado como $4\left(298-300{ }^{\circ} \mathrm{C} ; 55,0 \mathrm{mg}\right)$. A água mãe de
EGMAC-30-41 (1,0 g) foi submetida à cromatografia em coluna de gel de sílica usando como eluente inicial hexano:acetato de etila (1:1) e acrescentando acetato de etila e, em seguida, mistura de acetato de etila e metanol até metanol 100\%. Recolheram-se 30 frações $(250 \mathrm{~mL})$. As frações 5-7 foram submetidas à cromatografia de camada delgada preparativa e eluída duas vezes com a mistura $\mathrm{CHCl}_{3}: \mathrm{CH}_{3} \mathrm{OH}(8: 2)$. Uma mancha observada mediante luz UV foi marcada e raspada. A substância foi separada da sílica por filtração em funil de Hirsch lavando várias vezes com acetato de etila. Obteve-se um material pastoso que foi identificado como 5 (15,0 mg). A fração EGMAC-72-85 (1,2 g) foi submetida à cromatografia de coluna em gel de sílica utilizando como fase móvel $\mathrm{CHCl}_{3}: \mathrm{MeOH}(1: 1)$, acrescentando metanol até metanol $100 \%$. As frações 5-11 (130,0 mg) desta coluna foram reunidas após análise por cromatografia de camada fina, filtradas em coluna com gel de sílica, eluída com $\mathrm{CHCl}_{3}: \mathrm{MeOH}(9: 1)$ e obteve-se um material pastoso identificado como $\mathbf{6}(20,0 \mathrm{mg})$.

3,4,4',7-tetraidroxiflavana (Rel-2R,3S,4S-leucoguibourtinidina, 6)

RMN ${ }^{1} \mathrm{H}\left(200 \mathrm{MHz}, \mathrm{D}_{3} \mathrm{COD}\right): 3,87$ (dd, $J_{1}=9,8 \mathrm{~Hz}$ e $J_{2}=3,3$ $\mathrm{Hz}$; H-3,) 4,57 (d, $J=3,3 \mathrm{~Hz}$; H-4), 4,98 (d, $J=9,8 \mathrm{~Hz}, \mathrm{H}-2$ ), 6,23 (sl, H-8), 6,37 (d, $J=8,0 \mathrm{~Hz}$; H-6), 6,77 (d, $J=8,5, \mathrm{H}^{3} 3^{\prime}, 5$ ') , 7,10 (d, $J=8,0 \mathrm{~Hz}$; H-5), 7,25 (d, $\left.J=8,5, \mathrm{H}-2^{\prime}, 6^{\prime}\right)$; $\mathrm{RMN}^{13} \mathrm{C}(50,3 \mathrm{MHz}$, D $\mathrm{COD}): 69,1$ (C-4), 73,5 (C-3), 79,1 (C-2), 104,7 (CH-8), 110,3 (CH-6), 117,3 (CH-3',5'), 118,3 (C-10), 130,7 (C-1'), 131,5 (CH2',6'), 134,0 (C-5), 158,0 (C-9), 159,9 (C-7), 161,3 (C-4').

\section{AGRADECIMENTOS}

À CAPES, FAPERJ e ao CNPq pelas bolsas e auxílios concedidos e ao LCQUI-CCT-UENF pelos espectros a $400 \mathrm{MHz}$.

\section{REFERÊNCIAS}

1. http://mobot.mobot.org/cgi-bin/serch_vast;http://pt.wikipedia.org/wiki/ Schizolobium_parahyba, acessadas em Outubro 2007

2. Pesquisa Google, acessada em Outubro 2007; Rodrigues, W. A.; XLVIII Congresso Nacional de Botânica, Crato, Brasil, 1997.

3. Lorenzi, H.; Árvores Brasileiras-Manual de Identificação e Cultivo de Plantas Abóreas Nativas do Brasil, Ed. Plantarum: Nova Odessa, 1998, vol. 1, 2ª ed.; Rizini, C. T.; Árvores e Madeiras Úteis do Brasil, Edgar Blucher: São Paulo, 1986, p. 133.

4. Zawadzki-Baggio, S. F.; Sierakowski, M. R.; Corrêa, J. B. C.; Reicher, F. A.; Carbohydr. Res. 1992, 223, 265; Ganter J. L. M. S.; Heyraud, A.; Petkowicz, C. L. O.; Rinaudo, M.; Reicher, F.; Int. J. Biol. Macromol. 1995, 17, 13; Franco, T. T.; Rodrígues, N. R.; Serra, G. E.; Panegassi, V. R.; Buckeridge, M. S.; J. Chromatogr., B: Anal. Technol. Biomed. Life Sci. 1996, 680, 255.

5. Bresolin, T. M. B.; Sander, P. C.; Reicher, F.; Sierakowski, M. R.; Rinaudo, M.; Ganter, J. L. M. S.; Carbohydr. Polym. 1997, 33, 131.

6. Petkowicz, C. L. O.; Sierakowskil, J. R.; Ganter, J. L. M. S.; Reicher, F.; Phytochemistry 1998, 49, 737; Petrowicz, C. L. O.; Schaefer, S.; Reicher, F.; Carbohydr. Polym. 2007, 69, 659.

7. Luns, A. M.; Tese de Doutorado, Universidade Federal Rural do Rio de Janeiro, Brasil, 2004.

8. Nascimento, I. A. do; Dissertação de Mestrado, Universidade Federal Rural do Rio de Janeiro, Brasil, 2003.

9. Nascimento, I. A. do; Gomes, M. S. da Rocha; Carvalho, M. G. de; Carvalho, A. G. de; Rev. Univ. Rural, Ser. Cienc. Ex. e da Terra 2003, 22,81 .

10. Sallaud, C.; El-Turk, J.; Breda, C.; Buffard, D.; Kosakm, I. de; Esnaut, R.; Kondrosi, A.; Plant Sci. 1995, 109, 179; Hegnauer, R.; GrayerBarkmeijer, R. J.; Phytochemistry 1993, 34, 3.

11. Redmond, J. W.; Batley, M.; Djordjevic, M. A.; Innes, R. W.; Kuempel, P. L.; Rolfe, B. G.; Nature 1986, 323, 632. 
12. Chauarasia, N.; Wichtl, M.; J. Nat. Prod. 1987, 50, 881; Dutra, N. N.; Alves, H. de M.; Carvalho, M. G. de; Braz-Filho, R.; Quim. Nova 1992, 15, 10.

13. Yoshikawa, M.; Morikawa, T.; Xu, F.; Ando, S.; Matsuda, H.; Heterocycles 2003, 60, 1787.

14. Woo, W. S.; Kang, S. S.; Seligmann, L.; Chari, V. M.; Wagner, H.; Tetrahedron Lett. 1980, 21, 4255.

15. Haruna, M.; Koube, T.; Ito, K.; Murata, H.; Chem. Pharm. Bull. 1982, $30,1525$.

16. Hashimoto, Y.; Ozawa, S.; Sasaya, T.; Mokuzai Gakkaishi 1994, 40, 549.

17. Markham, K. R.; Mabry, T. J.; Tetrahedron 1968, 24, 823.

18. Wong, E.; Biochim. Biophys. Acta 1965, 111, 358; Wong, E.; Mortimer, P. I.; Geissman, T. A.; Phytochemistry 1965, 4, 89.

19. Ingham, J. L.; Z. Naturforsch., C: J. Biosci. 1978, 33, 146.

20. Wong, E.; Francis, C. M.; Phytochemistry 1968, 7, 2123.

21. Adinarayana, D.; Syamasundar, K. V.; Seligmann, O.; Wagner, H.; Z. Naturforsch., C: J. Biosci. 1982, 37, 145.

22. Hillis, W. E.; Inoue, T.; Phytochemistry 1966, 5, 483.
23. Silva, C. J. da; Oliveira, M. C. C. de; Carvalho, M. G. de; Resumos da 23a Reunião Anual da Sociedade Brasileira de Química, Poços de Caldas, Brasil, 2000.

24. Koshino, H.; Masaoka, Y.; Ichihara, A.; Phytochemistry 1993, 33, 1075.

25. Ayabe S. I.; Kobayashi, M.; Hikichi, M.; Matsumoto, K.; Furuya, T.; Phytochemistry 1980, 19, 2179.

26. Markham, K. R.; Mabry, T. J.; Swift Jr., W. T.; Phytochemistry 1970, 9 , 2359.

27. Jung, M. J.; Kang, S. S.; Jung, H. A.; Kim, G. J.; Choi, J. S.; Arch. Pharmacal Res. 2004, 27, 593.

28. Tomás-Barberán, F. A.; García-Grau, M. M.; Tomás-Lorente, F.; J. Agric. Food Chem. 1991, 39, 255.

29. Steynberg, J. P.; Ferreira, D.; Roux, D. G.; Tetrahedron Lett. 1983, 24, 4147.

30. Steynberg, J. P.; Brant, E. V.; Ferreira, D.; Hemingway, R. W.; J. Chem. Soc., Perkin Trans. 1997, 1, 1943.

31. Ali, M.; Bhutani, K. K.; Gupta, D. K.; Fitoterapia 1997, 68, 82.

32. Pauletti, P. M.; Bolsani, V. da S.; Young, M. C. M.; Quim. Nova 2003, 26, 641. 